\title{
Did Residential Racial Segregation in the U.S. Really Increase? An Analysis Accounting for Changes in Racial Diversity
}

\author{
Benjamin Elbers \\ Columbia University \\ be2239@columbia.edu \\ Current version · Replication materials
}

July 13, 2021

\begin{abstract}
A recent study by UC Berkeley's Othering \& Belonging Institute (Menendian et al. 2021) came to an astonishing conclusion: Of large metropolitan areas in the U.S., $81 \%$ have become more segregated over the period 1990-2019. This finding contradicts the recent sociological literature on changes in segregation in the U.S., which has generally found that racial residential segregation has slowly declined since the 1970s, especially between Blacks and Whites. The major question then is: What accounts for this difference? This paper answers this question in two parts. First, it shows that the preferred segregation measure of the Berkeley study, the "Divergence Index" (Roberto 2015), is identical to the Mutual Information Index $M$ (Mora and Ruiz-Castillo 2009, 2011; Theil and Finizza 1971), a measure that is mechanically affected by changes in racial diversity. Given that the U.S. has become more diverse over the period 1990 to 2019, it is not surprising that this index shows increases in segregation. Second, by making use of a decomposition procedure developed in Elbers (2021), the paper shows that once the changes in segregation are decomposed into components that account for the changing racial diversity of the U.S., the findings are in line with the sociological literature. Residential racial segregation as a whole has declined modestly in most metropolitan areas of the U.S., although segregation has increased slightly when focusing on Asian Americans and Hispanics.
\end{abstract}

\section{Introduction}

A recent study by UC Berkeley's Othering \& Belonging Institute (Menendian et al. 2021, from here one referred to as the Berkeley study) came to an astonishing conclusion: Of 
large metropolitan areas in the U.S., $81 \%$ have become more segregated over the period 1990-2019. This finding contradicts most of the sociological literature on changes in segregation in the U.S., which has generally found that racial residential segregation has slowly declined since the 1970s (e.g., Charles 2003). The study was picked up by many major news outlets, such as Bloomberg, Forbes Time Magazine, and The Guardian. A small number of critical comments on the study were also published, namely in the form of an opinion piece in the Wall Street Journal (with a response from the authors) and a blog post.

The study raises a major question: What accounts for this difference in trends? This paper answers this question in two parts, and reconciles the findings of the Berkeley study with the sociological literature. First, it shows that the preferred segregation measure of the Berkeley study, the "Divergence Index" (Roberto 2015), is identical to the Mutual Information Index $M$ (Mora and Ruiz-Castillo 2009, 2011; Theil and Finizza 1971), a measure that is severely affected by changes in racial diversity. The higher the racial diversity of a metropolitan area, the higher the $M$ index. Given that the U.S. has become more diverse over the period 1990 to 2019, it is not surprising that the Berkeley study finds an increase in segregation. In the methodological literature on segregation, this issue of "margin dependency" is central as it allows making comparisons across metropolitan areas and over time even when the racial diversity differs; however, this issue is not discussed in the Berkeley study. The methodological discussion also shows that the advantages of the $M$ index are somewhat overstated in both the Berkeley study and Roberto's working paper (2015): The more commonly used $H$ index is identical to the $M$ index except for division by a scaling factor. As will be shown below, both indices measure segregation, as well as relative differences in diversity.

In a second step, by making use of decomposition procedure developed in Elbers (2021), the paper shows that once the changes in segregation are decomposed into components that account for the changing racial diversity of the U.S., the findings are in line with the sociological literature. Residential racial segregation as a whole has declined modestly in most metropolitan areas of the U.S., although segregation has increased slightly when focusing on segregation between Whites and Asian Americans and Whites and Hispanics. Notably, wherever there are declines in segregation, these have been rather small: In most metropolitan areas, despite years of decline, segregation is still exceptionally high. The analysis shows that it is important to either choose an index that is designed to facilitate comparisons across metropolitan areas and across time (such as the $H$ or the $D$ index), or to decompose changes in segregation to adjust for changing racial diversity. ${ }^{1}$

In the next section, it is shown that the Divergence Index is identical to the Mutual Information Index $M$, and the issue of the $M$ 's margin-dependency is discussed. The

\footnotetext{
${ }^{1}$ There is a parallel here to the literature on school segregation: Some studies on changes in school racial segregation have also used a margin-dependent index (in this case, exposure indices) and came to the conclusion that school segregation has increased (Orfield et al. 2002). Analyses that correct for the fact that exposure indices are impacted by changing racial diversity come to the opposite conclusion, namely that school racial segregation has slightly declined (Fiel 2013).
} 
following section replicates the findings of the Berkeley study and decomposes the differences in segregation for each metropolitan area into components that account for this margin dependency. The paper concludes that, while the $M$ index is useful, researchers need to be careful when using this index to make comparisons across space and time.

\section{The Divergence Index is Mutual Information}

Any segregation index summarizes a table of racial group counts across spatial units to a single number. The unit of analysis is usually a metropolitan area, with the spatial units being Census tracts, blocks, or another definition of neighborhood. Let there be $U$ spatial units in the metropolitan area, for each which we obtain counts for $G$ racial groups. Let $t_{u g}$ be the number of people of racial group $g$ in neighborhood $u$. Let $T=\sum_{u=1}^{U} \sum_{g=1}^{G} t_{u g}$ be the total population in the metropolitan area. Then define the cell probabilities as $p_{u g}=t_{u g} / T$ and the marginal probabilities as $p_{u}=\sum_{g=1}^{G} p_{u g}$ and $p_{g} .=\sum_{u=1}^{U} p_{u g}$. Lastly, define the conditional probabilities $p_{u \mid g}=p_{u g} / p_{\cdot g}$ and $p_{g \mid u}=p_{u g} / p_{u}$. Note the difference between $p_{g}$. and $p_{g \mid u}$ : the first describes the overall proportion of racial group $g$ in the metropolitan area (the marginal probability, as this is a column sum in the contingency table), while $p_{g \mid u}$ describes the proportion of racial group in neighborhood $u$. Intuitively, segregation is high when the proportions $p_{g \mid u}$ differ strongly from the overall proportions, $p_{g}$. In the following, bolded letters refer to vectors of probabilities, for instance, $\mathbf{p}_{g} .=\left(p_{1 .}, p_{2}, \ldots, p_{G}.\right)$.

The idea of the Divergence Index is then as follows. For each neighborhood, define a quantity $L_{u}$ that is the Kullback-Leibler divergence of the distributions $\mathbf{p}_{g \mid u}$ and $\mathbf{p}_{g}$, i.e.

$$
L_{u}=\sum_{g=1}^{G} p_{g \mid u} \log \frac{p_{g \mid u}}{p_{\cdot g}} .
$$

This is a measure of how strongly neighborhood $u$ 's racial composition deviates from the overall racial composition of the metropolitan area. If these are identical, then $L_{u}=0$. I refer to this measure as $L_{u}$, because Mora and Ruiz-Castillo $(2009,2011)$ refer to this measure as "local segregation." Alonso-Villar and del Río (2010) also call this measure "local segregation" and derive $L_{u}$ axiomatically. Much earlier, Theil and Finizza (1971, p. 190) have derived this quantity for the two-group case and called it an "information expectation." To arrive at an overall measure of segregation for the entire metropolitan area, the $L_{u}$ are then weighted by the size of each neighborhood, i.e.

$$
M=\sum_{u=1}^{U} p_{u} \cdot L_{u}=\sum_{u=1}^{U} p_{u} \cdot\left(\sum_{g=1}^{G} p_{g \mid u} \log \frac{p_{g \mid u}}{p_{\cdot g}}\right) .
$$

While each $L_{u}$ is indeed a Kullback-Leibler divergence, this is not true for the overall index $M$. This index is identical to mutual information, not divergence. To see this, 
use the identity $p_{g \mid u}=p_{u g} / p_{u}$ :

$$
\begin{aligned}
M & =\sum_{u=1}^{U} p_{u}\left(\sum_{g=1}^{G} p_{g \mid u} \log \frac{p_{g \mid u}}{p_{\cdot g}}\right) \\
& =\sum_{u=1}^{U} p_{u} \cdot\left(\sum_{g=1}^{G} \frac{p_{u g}}{p_{u \cdot}} \log \frac{p_{u g}}{p_{\cdot g} p_{u} \cdot}\right) \\
& =\sum_{u=1}^{U} \sum_{g=1}^{G} p_{u g} \log \frac{p_{u g}}{p_{\cdot g} p_{u} .}
\end{aligned}
$$

Arguably, this index is more usefully referred to as the Mutual Information Index, as this is what this quantity represents: it is the mutual information of the joint distribution of the racial groups and the spatial units. The use of mutual information as a segregation index has been described first by Theil and Finizza (1971) and in much more detail by Mora and Ruiz-Castillo (2009, 2011), who introduced the symbol $M$.

Some other useful identities can be derived for mutual information. For this, it is useful to define the entropy of a distribution as $E(\mathbf{p})=-\sum p_{i} \log p_{i}$, where the sum extends over all elements of the vector $\mathbf{p}$. Then, the following five identities can be derived, all of which are useful in understanding the use of mutual information as a segregation index:

$$
\begin{aligned}
M & =\sum_{u=1}^{U} \sum_{g=1}^{G} p_{u g} \log \frac{p_{u g}}{p_{u \cdot p} \cdot g} \\
& =\mathrm{E}\left(\mathbf{p}_{u \cdot}\right)+\mathrm{E}\left(\mathbf{p}_{\cdot g}\right)-\mathrm{E}\left(\mathbf{p}_{u g}\right) \\
& =\sum_{u=1}^{U} p_{u \cdot}\left(\sum_{g=1}^{G} p_{g \mid u} \log \frac{p_{g \mid u}}{p_{\cdot g}}\right) \\
& =\sum_{g=1}^{G} p_{\cdot g}\left(\sum_{u=1}^{U} p_{u \mid g} \log \frac{p_{u \mid g}}{p_{u \cdot}}\right) \\
& =\sum_{u=1}^{U} p_{u \cdot}\left(\mathrm{E}\left(\mathbf{p}_{\cdot g}\right)-\mathrm{E}\left(\mathbf{p}_{g \mid u}\right)\right) \\
& =\sum_{g=1}^{G} p_{\cdot g}\left(\mathrm{E}\left(\mathbf{p}_{u \cdot}\right)-\mathrm{E}\left(\mathbf{p}_{u \mid g}\right)\right)
\end{aligned}
$$

Any of these six forms can be used to compute the $M$ index. Because mutual information is symmetric, identities are also included that allow decomposition of the index in terms of racial groups. For instance, the fourth form writes the index as a weighted sum of local segregation scores (or divergence scores) for each racial group. 
The identities also show that, while it is true that this index measures the deviations of each neighborhood's racial distribution from the overall racial distribution, it is also true that the index measures the difference between a neighborhood's diversity and the overall metropolitan area's diversity, as the fifth form shows. The identities show that the index measures both of these concepts at the same time.

With some exceptions, mutual information has not been used very much as a segregation index (or more generally, as a measure of association in contingency tables). ${ }^{2}$ However, the adoption of a closely related index, Theil's Information Index $H$, has been increasing. The construction of the $H$ index is intuitively illustrated in the case of entirely segregated cities, which also illuminates an important property of the $M$ index. By definition, in an entirely segregated city, every neighborhood contains only one racial group. Consider the following two small cities (of course, this could also represent the same city at two points in time), each of which has four neighborhoods (rows) and two racial groups (columns):

$$
\text { City A: }\left[\begin{array}{cc}
50 & 0 \\
50 & 0 \\
0 & 50 \\
0 & 50
\end{array}\right] \quad \text { City B: }\left[\begin{array}{cc}
50 & 0 \\
50 & 0 \\
0 & 10 \\
0 & 10
\end{array}\right]
$$

Each of these cities is completely segregated in the sense that there is no racial mixing within neighborhoods, and therefore, the Dissimilarity Index for both of these cities is 1. However, the $M$ index of city $\mathrm{A}$ is 0.69 , but the $M$ index of city $\mathrm{B}$ is 0.45 . Given that city $\mathrm{B}$ is maximally segregated, it is impossible for this city to obtain a higher $M$ index! This is an important property that users of the $M$ index need to be aware of, as it greatly impacts interpretation of the index. The simple example shows that the maximum value the $M$ index can reach is identical to $\mathrm{E}\left(\mathbf{p}_{\cdot g}\right)$, the diversity of the metropolitan area. Hence, the idea is to "normalize" the $M$ index by dividing by the entropy of the racial group distribution, i.e. $\mathrm{E}\left(\mathbf{p}_{\cdot g}\right)$, to obtain $H$ :

$$
H:=\frac{M}{\mathrm{E}\left(\mathbf{p}_{\cdot g}\right)} .
$$

Under certain conditions that are usually true in empirical settings (see endnote 3 in Elbers 2021), this division has the effect that the $H$ index ranges from 0 to 1 . In many settings, the $H$ index will usually be the more appropriate index compared to $M$, as it is easier to interpret, and we can still obtain useful local segregation measures for this index, as will be shown below.

\subsection{The Core Issue of the $M$ Index: Margin Dependency}

The $H$ index also has another advantage: It is much less margin-dependent than the $M$ index. The issue of margin-dependency is not discussed in the Berkeley study,

\footnotetext{
${ }^{2}$ Some exceptions are Alonso-Villar et al. (2012), DiPrete et al. (2017), Elbers et al. (2020), Frankel and Volij (2011), and Gradín et al. (2015).
} 
although it has been one of the major issues in the methodological literature of segregation indices. The problem of margin dependency refers to the fact that, although the association between racial groups and certain neighborhoods has not changed, the index changes its value. Put more directly, the index can be affected by changes in diversity (racial group margin dependency) and/or by changes in the relative sizes of neighborhoods (spatial unit margin dependency). In the segregation literature, it has long been realized that it is necessary to choose indices that are independent of the diversity of a metropolitan area, especially when comparisons over time and/or across metropolitan areas are desired. The example discussed in the previous section has demonstrated that this margin dependency can be of serious concern when comparing cities: Although both cities are completely segregated, one city has a index that is almost 50\% higher than the other. More examples are provided in Elbers (2021).

Given that the $M$ index is a useful measure of segregation in the cross section-see the overview of decomposition properties in Mora and Ruiz-Castillo (2009, 2011) for a full appreciation-, it seems premature to entirely advocate against its use. However, users of the index need to be aware of the serious problems of interpretation that arise when using this index to make a judgement of whether one city has become more or less segregated over time, or whether one city is more segregated than another. One solution to this problem of making comparisons is a decomposition procedure that allows the researcher to decompose the change or difference in segregation into components that account for the margin dependencies of the index, and a component that captures whether the association between racial groups and neighborhoods has become stronger or weakened. Such a procedure is described in detail in Elbers (2021), and this procedure will be used to reassess the results of the Berkeley study below.

\subsection{Measures of Local Segregation}

Lastly, we take a closer look at the local segregation scores of both the $M$ and the $H$ index that capture the contribution of each neighborhood. This question is tangential to the Berkeley study, because the study does not make use of these quantities: Only aggregate $M$ indices are presented, which is quite reasonable given that each metropolitan area consists of hundreds or thousands of Census tracts. Nonetheless, the decomposition of the $M$ and $H$ indices into local segregation (or divergence) scores is conceptually appealing when studying, for instance, a single metropolitan area. For the $M$ index, we have already seen that we can write $M=p_{u} . L_{u}$. For the $H$ index, we can equivalently define

$$
L_{u}^{(H)}:=\frac{L_{u}}{\mathrm{E}(\mathbf{p} \cdot g)},
$$

such that $H=p_{u} \cdot L_{u}^{(H)}$. The measure $L_{u}^{(H)}$ is a scaled Kullback-Leibler divergence. Hence, we can also write the $H$ index as a weighted sum of local segregation scores. (Note, however, that unlike the aggregate $H$ index, the scores $L_{u}^{(H)}$ are not restricted 
to the range from zero to one). The only difference between $L_{u}$ and $L_{u}^{(H)}$ is the division by the racial group entropy.

Roberto (2015) rightly points out that the measure $L_{u}$ is more reasonable than a measure involving $\mathrm{E}\left(\mathbf{p}_{\cdot g}\right)-\mathrm{E}\left(\mathbf{p}_{g \mid u}\right)$, the differences in diversity, when the goal is to capture segregation at a local level. ${ }^{3}$ However, as has been shown, the definition of $H$ is no more or less tied to the differences in entropy, $\mathrm{E}\left(\mathbf{p}_{\cdot g}\right)-\mathrm{E}\left(\mathbf{p}_{g \mid u}\right)$ than the $M$ index. Hence, the critique that the $H$ index "measures relative homogeneity, and it is misleading to interpret it as a measure of segregation" Roberto (2015, p. 6), is not valid: While the $H$ index does measure entropy differences, it also measures divergence. The same statement is hence also true for the $M$ index. More generally, any critique that is leveled against the $H$ index will also likely be valid for the $M$ index, given that the only difference between the two indices is the division by a scaling factor.

\section{Replication and Decomposition Results}

\subsection{Data}

In this section, I replicate the results of the Berkeley study. The data used are the decennial Censuses of 1990, 2000, and 2010, as well as the American Community Survey (ACS) estimates 2015-2019, all of which are obtained from NHGIS (Manson et al. 2021). The metropolitan area definitions used are the Core Based Statistical Area definitions for 2013. Only metropolitan areas with a population of more than 200,000 in 2019 are retained in the analysis, yielding 220 metropolitan areas for analysis. (The Berkeley study reports results for 209 metropolitan areas.) The spatial units are Census tracts, and the racial groups are non-Hispanic Whites, non-Hispanic Blacks, non-Hispanic Asians, non-Hispanic Native American, and Hispanics. Individuals who are classified as another race, or as two or more races, are classified as "Other".

One problematic aspect of this dataset that is not discussed in the Berkeley study is the use of the ACS estimates for 2019. Compared to Census data, studies have shown that the use of ACS data inflates segregation estimates. Furthermore, the ACS estimates are associated with margins of error, which especially for small racial groups can be quite large. The present analysis makes no attempt to rectify the problem of sampling bias or to take into account the margins of error.

All computations were done using $\mathrm{R}$ ( $\mathrm{R}$ Core Team 2021) and the R package "segregation".

\footnotetext{
${ }^{3}$ Roberto (2015) cites Theil and Finizza (1971), suggesting that they proposed $\mathrm{E}\left(\mathbf{p}_{\cdot g}\right)-\mathrm{E}\left(\mathbf{p}_{g \mid u}\right)$ as a measure of local segregation. As has been shown above, they in fact proposed the divergence measure. I am not aware of any work that has suggested to use the differences in entropy as a local measure of segregation.
} 


\begin{tabular}{lccrl}
\hline Groups & 1990 & 2019 & Difference & Difference \% \\
\hline D Index & & & & \\
Asian-White & 0.377 & 0.463 & 0.086 & 22.8 \\
Black-White & 0.557 & 0.523 & -0.034 & -6.0 \\
$\quad$ Hispanic-White & 0.321 & 0.411 & 0.090 & 28.2 \\
H Index & & & & \\
Asian-White & 0.089 & 0.160 & 0.071 & 79.4 \\
Black-White & 0.293 & 0.246 & -0.047 & -16.1 \\
Hispanic-White & 0.080 & 0.152 & 0.072 & 90.6 \\
Multigroup & 0.217 & 0.170 & -0.047 & -21.7 \\
M Index & & & & \\
Asian-White & 0.007 & 0.025 & 0.018 & 266.8 \\
Black-White & 0.093 & 0.091 & -0.002 & -1.7 \\
Hispanic-White & 0.011 & 0.051 & 0.040 & 362.8 \\
Multigroup & 0.131 & 0.164 & 0.033 & 25.3 \\
\hline
\end{tabular}

Table 1: Results for different indices for the median metropolitan area

\subsection{Replication}

For the replication, I compute eleven segregation indices. Using the definitions of $H$ and $M$ above, I compute multigroup, Black-White, Hispanic-White, and AsianWhite segregation. I also compute segregation using the index of dissimilarity $D$. This index only allows pairwise comparisons. As there are relatively few details given in the Berkeley study regarding the construction of the dataset, some of the design decision may differ from the original study. However, the correlation obtained between the $M$ indices reported in the study and the indices calculated in this replication are 0.997. Therefore, the replication can be considered successful.

Table 1 shows the median index values for 1990 and 2019, together with their difference. In the median metropolitan area, the Black-White Dissimilarity index declined by about $6 \%$, while Hispanic-White segregation and Asian-White segregation increased by about $28 \%$ and $22 \%$, respectively. For the $H$ index, the comparative index values are lower (note both the $D$ and the $H$ are standardized to the range from zero to one), but the trends are in the same direction: A decline in Black-White segregation (which nonetheless remains high), and large increases in Hispanic-White and Asian-White segregation. The same trends are again found for the $M$ index, although the increases are much larger in percentage terms for Hispanic-White and Asian-White segregation.

Perhaps the most important results are the multigroup indices, which are the focus of the Berkeley study. Here, the $H$ and the $M$ index contradict each other strongly: While, according to the $H$ index, multigroup segregation declined by about $22 \%$, it increased by about $25 \%$ according to the $M$ index. The authors of the Berkeley study argue that the $M$ index is a superior measure (and hence, that those results that 


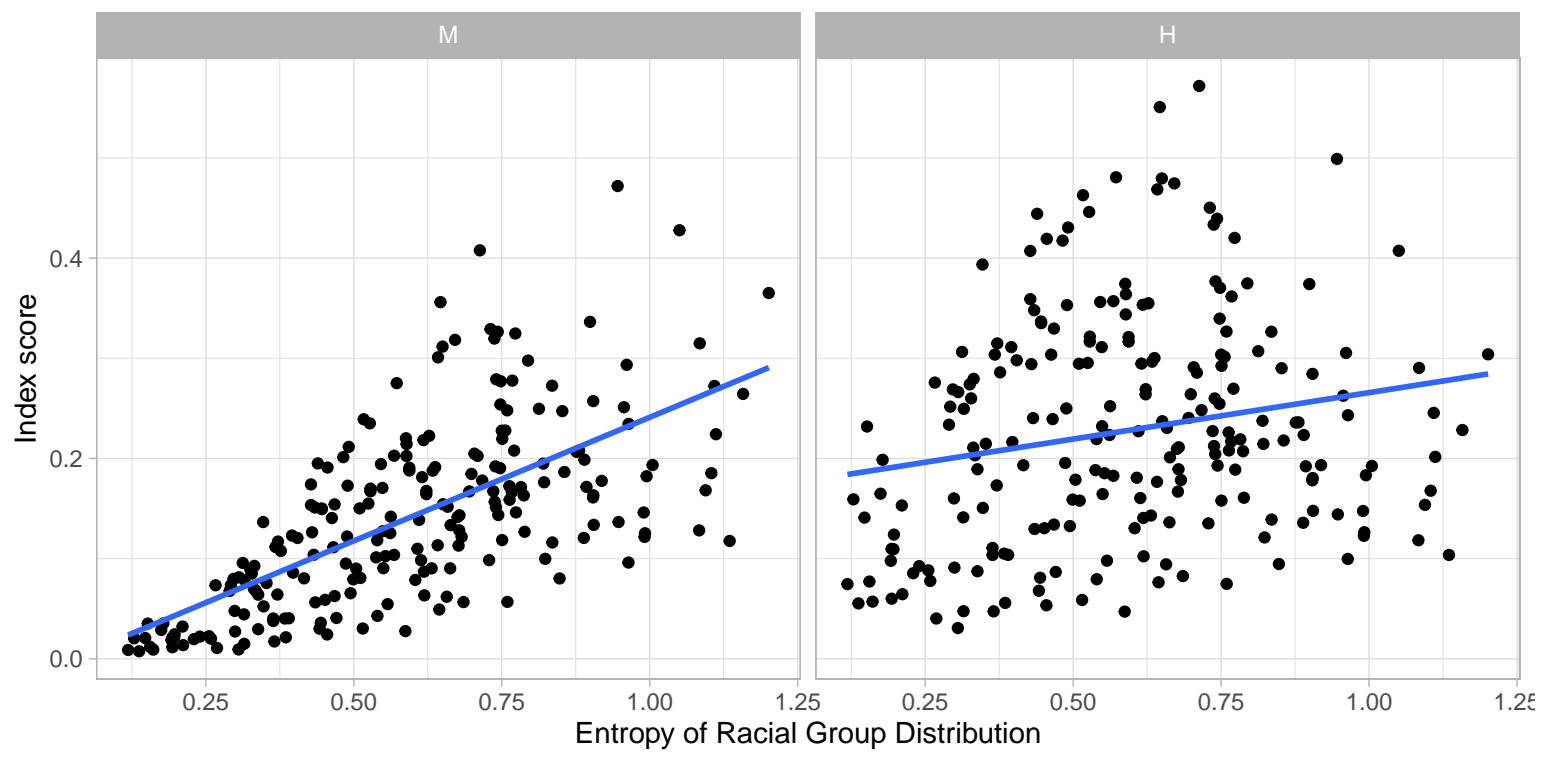

Figure 1: Scatter plots of racial group entropy and $M / H$ indices

show increases in segregation should be trusted), however, as we have seen above, the difference between the $M$ and the supposedly-flawed $H$ index is minor-it only consists of a division by the racial group entropy. The fact that the results for the $M$ and the $H$ index so strongly diverge suggests that changes in diversity are strongly influencing the increase in the $M$ index. A decomposition analysis in the next section shows that this is indeed the case.

Some empirical evidence of this fact is also presented in Figure 1. This figure shows two scatter plots, where the x-axis shows the entropy of the racial group distribution (i.e., a measure of the metropolitan area's diversity), and the y-axis shows the index score for the $M$ index (left panel) and the $H$ index (right panel). Clearly, for the $M$ index, there is a strong dependency between the index value and the diversity of the metro area. For the $M$, the correlation is 0.66 , while for the $H$, the correlation is only 0.20. This figure shows empirically what has been demonstrated above theoretically: the $M$ index is determined, to a some extent, by racial diversity in the metropolitan area. A metropolitan area with an entropy of, say, 0.25 , can only reach an $M$ index value of 0.25 , even if it is completely segregated. Because the $H$ index partially accounts for these differences in diversity, the relationship is far less strong.

The Berkeley study also argues that "the prevailing narrative around gradual residential integration relies primarily on a measure of segregation that is misleading and flawed in many ways, but chiefly because it focuses on the binary of Black-white segregation." Multigroup measures are indeed important to measure the segregation of all racial groups from each other simultaneously. However, pairwise indices remain instructive in learning about the racial groups that are "most responsible" for increases or decreases in segregation. Table 2 shows correlations for 2010 for all eleven indices. 


\begin{tabular}{|c|c|c|c|c|c|c|c|c|c|c|c|}
\hline & \multicolumn{2}{|c|}{ Multigroup } & \multicolumn{3}{|c|}{ Black-White } & \multicolumn{3}{|c|}{ Hispanic-White } & \multicolumn{3}{|c|}{ Asian-White } \\
\hline & $\mathrm{M}$ & $\mathrm{H}$ & M & $\mathrm{H}$ & $\mathrm{D}$ & $\mathrm{M}$ & $\mathrm{H}$ & $\mathrm{D}$ & $\mathrm{M}$ & $\mathrm{H}$ & $\mathrm{D}$ \\
\hline \multicolumn{12}{|c|}{ Multigroup } \\
\hline M & 1.00 & 0.89 & 0.48 & 0.52 & 0.49 & 0.42 & 0.21 & 0.17 & 0.02 & 0.45 & 0.38 \\
\hline $\mathrm{H}$ & 0.89 & 1.00 & 0.75 & 0.45 & 0.44 & 0.40 & 0.33 & 0.31 & 0.26 & 0.41 & 0.48 \\
\hline \multicolumn{12}{|c|}{ Black-White } \\
\hline M & 0.48 & 0.75 & 1.00 & 0.17 & 0.19 & 0.22 & 0.29 & 0.34 & 0.47 & 0.17 & 0.42 \\
\hline $\mathrm{H}$ & 0.52 & 0.45 & 0.17 & 1.00 & 0.95 & 0.75 & 0.45 & 0.43 & 0.18 & 0.82 & 0.69 \\
\hline $\mathrm{D}$ & 0.49 & 0.44 & 0.19 & 0.95 & 1.00 & 0.88 & 0.43 & 0.43 & 0.20 & 0.88 & 0.79 \\
\hline \multicolumn{12}{|c|}{ Hispanic-White } \\
\hline M & 0.42 & 0.40 & 0.22 & 0.75 & 0.88 & 1.00 & 0.31 & 0.33 & 0.17 & 0.83 & 0.86 \\
\hline $\mathrm{H}$ & 0.21 & 0.33 & 0.29 & 0.45 & 0.43 & 0.31 & 1.00 & 0.97 & 0.78 & 0.59 & 0.62 \\
\hline $\mathrm{D}$ & 0.17 & 0.31 & 0.34 & 0.43 & 0.43 & 0.33 & 0.97 & 1.00 & 0.87 & 0.60 & 0.68 \\
\hline \multicolumn{12}{|c|}{ Asian-White } \\
\hline M & 0.02 & 0.26 & 0.47 & 0.18 & 0.20 & 0.17 & 0.78 & 0.87 & 1.00 & 0.38 & 0.61 \\
\hline $\mathrm{H}$ & 0.45 & 0.41 & 0.17 & 0.82 & 0.88 & 0.83 & 0.59 & 0.60 & 0.38 & 1.00 & 0.89 \\
\hline $\mathrm{D}$ & 0.38 & 0.48 & 0.42 & 0.69 & 0.79 & 0.86 & 0.62 & 0.68 & 0.61 & 0.89 & 1.00 \\
\hline
\end{tabular}

Table 2: Correlation between indices (using 2010 data)

Correlations between Black-White segregation indices and multigroup indices are high, because these two population groups account for a very large share of the U.S. population. For instance, the correlation between the multigroup $M$ and the Black-White $M$ index is 0.86 (for the $H$ index: 0.88). Hence, multigroup measures may not reveal as many new insights into segregation dynamics as often argued. In any way, this analysis shows that the differences between the Berkeley study and the existing sociological literature is not explained by the use of a multigroup index, but by the choice of margin-dependent segregation index.

It is also notable that the correlations among the pairwise indices are large: for Black-White segregation, the $M$ and $H$ correlate at 0.88 , the $M$ and $D$ at 0.75 , and the $H$ and $D$ at 0.95 . These correlations are also generally large for Hispanic-White and Asian-White segregation, and show that, while the $H$ and $M$ indices are in many ways preferable measures due to their decomposability, the results obtained using the $D$ index will in most cases not differ wildly from those of the information-theoretic measures. This is not surprising given that all these measures are based on an evenness conceptualization of segregation (Massey and Denton 1988).

\subsection{Decomposition}

The decomposition method developed in Elbers (2021) requires spatially harmonized data. This means that it is only possible to decompose changes over time, but not 
changes between metropolitan areas. NHGIS provides spatially harmonized data based on 2010 Census tracts, such that data from the Censuses of 1990 and 2000 is crosswalked to 2010 Census tract geographies. As some Census tracts are divided or merged for new Censuses, this process introduces uncertainties into the estimates. Therefore, I compute the segregation indices that were shown in the previous section also for the harmonized data. The correlations between the segregation indices using the unharmonized and the harmonized data range from 0.96 to 0.99 . Hence, the uncertainties introduced during the harmonization process are of only minor importance for the segregation results.

The decomposition method decomposes the change in segregation into three components: one component accounts for changes in the racial group distribution-i.e., changes in diversity - , one component accounts for changes in the Census tract distributionfor instance, general population shifts to the suburbs-, and a last component accounts for changes in the association between Census tracts and racial groups, which could be termed "pure segregation" (this term is called "structural" in Elbers 2021). In the methodological literature on segregation, the consensus is that differences in diversity should not influence the level of the index. Hence, the racial group marginal component should not be interpreted as a substantive change in segregation. The interpretation of the Census tract marginal component is more ambiguous: If the population grew especially in segregated neighborhoods, then this component should also be interpreted as a change in segregation.

Table 3 shows the decomposition results for five examples metropolitan areas. The New York, Chicago, Los Angeles, and Dallas metropolitan areas are the four largest metro areas in the U.S. as of 2019, and the Fayetteville-Springdale-Rogers metropolitan area was chosen because it shows the largest increase in the $M$ index. In New York, Chicago, and Los Angeles, the $M$ index declined. The decomposition reveals that if only the diversity of the cities would have changed, without altering the association between neighborhoods and racial groups, then the $M$ index would have increased in New York by 0.036 and in Chicago by 0.058 . This shows that in these cities, the changing racial diversity strongly impacts the $M$ index. Importantly, the association component is negative in all the three large metropolitan areas, showing that the association between neighborhoods and racial groups in fact weakened, and segregation therefore declined.

The Dallas metropolitan area is representative of a pattern that occurs in about $45 \%$ of the U.S. metropolitan areas: the racial group marginal positive is positive, but the association component is negative. The overall increase in the $M$ index in Dallas is a mechanical consequence of the increasing diversity of the metropolitan area, and should not be interpreted as an increase in segregation. The case of Fayetteville-SpringdaleRogers is also instructive: Of the large increase in the $M$ index $(+0.124)$, more than $80 \%$ are accounted for by the changing diversity of the metropolitan area. While the association component also increased, this increase was much smaller than the large increase in the $M$ index might suggest.

Table 3 shows results only for five metropolitan areas, so Figure 2 shows histograms of the Difference in $M$, as well as the three decomposition components for all metropolitan areas. The differences in $M$ are indeed mostly positive, confirming the findings 


\begin{tabular}{lrrrrr}
\hline variable & New York & Los Angeles & Chicago & Dallas & Fayetteville \\
\hline Outcomes & & & & & \\
$\quad$ Multigroup M 1990 & 0.429 & 0.372 & 0.474 & 0.265 & 0.021 \\
$\quad$ Multigroup M 2019 & 0.424 & 0.351 & 0.427 & 0.286 & 0.145 \\
$\quad$ Difference in M & -0.005 & -0.022 & -0.047 & 0.021 & 0.124 \\
Decomposition & & & & & \\
$\quad$ Racial group margins & 0.035 & -0.006 & 0.055 & 0.058 & 0.102 \\
$\quad$ Census tract margins & 0.006 & -0.011 & -0.020 & -0.032 & 0.001 \\
$\quad$ Association & -0.046 & -0.004 & -0.081 & -0.004 & 0.021 \\
\hline
\end{tabular}

Table 3: Decomposition results for four example metropolitan areas

of the Berkeley study. However, in $92 \%$ of the metropolitan areas, the racial group marginal component was also positive: This is clearly an effect of the increasing diversity of the U.S., which the $M$ index captures, but it should not be taken as evidence of increasing segregation. The Census tract marginal component is more concentrated, but was negative for $80 \%$ of metropolitan areas, which means that in those areas, the population shifted from more segregated to less segregated neighborhoods. Lastly, the association component shows mixed results: The distribution is almost symmetric and centered above zero. Hence, the association between racial groups and neighborhoods increased in roughly half, and decreased in roughly half of the metropolitan areas of the U.S.

If we take both the Census tract marginal component and the association component as the outcomes of interest, then segregation declined in about $61 \%$ of the metropolitan areas of the U.S. This last estimate is, in effect, an estimate of segregation change that controls for changes in diversity. This estimate is highly correlated (0.8) with the simple difference in multigroup $H$ indices, indicating that a similar conclusion would have been reached using the $H$ index. ${ }^{4}$

\section{Conclusion}

This paper has made five major points:

1. The Divergence Index is identical to the Mutual Information index $M$. Given that the aggregate measure is identical to mutual information and not Kullback-Leibler divergence, this term is more appropriate. The $M$ index can be decomposed into local segregation, or divergence scores. A weighted average of these scores yields the aggregate $M$ index. A similar decomposition can be obtained for the closelyrelated $H$ index.

\footnotetext{
${ }^{4}$ While the $H$ is less margin-dependent than the $M$, it is still slightly margin-dependent. This is why the decomposition involving the $M$ index is somewhat more precise, and why the correlation is not perfect.
} 


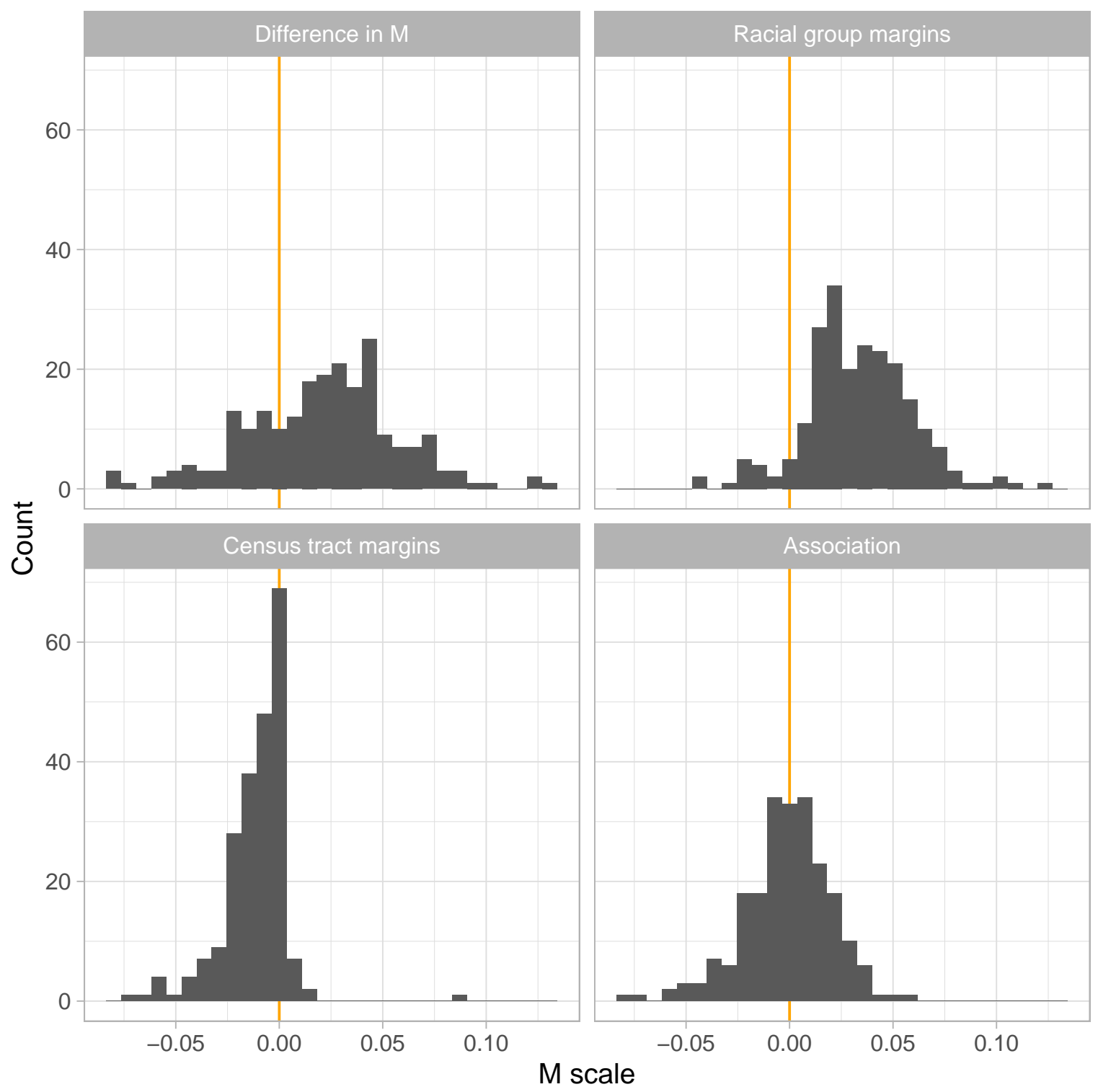

Figure 2: Histograms of decomposition results for all metro areas 
2. The $M$ index is mechanically influenced by the diversity of the metropolitan area. Notably, a metropolitan area with higher diversity can also obtain a higher index score. Of course, this doesn't mean that for any given two metropolitan areas, the one with more diversity will always have a higher $M$ index. The results show, however, that the relationship is not only theoretical, but that diversity and the $M$ index are closely related empirically. Therefore, the raw index values should not be used to rank metropolitan areas, or to compare metropolitan areas over time.

3. If a comparison over time is desired, a decomposition method should be employed such that the part of the change in the $M$ index that is brought about by changing diversity can be accounted for.

4. Once the differences in diversity are accounted for, the results for the $M$ index no longer differ very much from results obtained using the $D$ or the $H$ index. This is not surprising given that the all of these indices are variants of "evenness" measures of segregation (Massey and Denton 1988).

5. Lastly, while the $M$ index has a number of advantages in terms of decomposition capabilities (Mora and Ruiz-Castillo 2009, 2011), many researchers are well-served by Theil's $H$ index (see also Reardon and Firebaugh 2002). Unlike the $M$ index, it is scaled from zero to one and partially accounts for differences in diversity, so it is more appropriate when comparing and ranking metropolitan areas.

Empirically, the analysis has shown that segregation declined in most metropolitan areas of the U.S. However, the decline is not uniform (for instance, segregation between Hispanics and Whites and Asians and Whites has increased), and it has been rather small. Many U.S. metropolitan areas remain severely segregated, and it is far too early to proclaim the "end of the segregated century" (Glaeser and Vigdor 2012).

\section{Appendix}

Some of these proofs are also provided by Mora and Ruiz-Castillo (2009, 2011). All of these proofs are presented as algebraic identities, but they are all following from basic information-theoretic principles, so they are also defined for random variables.

First, write $M$ as the sum of three entropies: 


$$
\begin{aligned}
M & =\sum_{u=1}^{U} \sum_{g=1}^{G} p_{u g} \log \frac{p_{u g}}{p_{\cdot g} p_{u}} \\
& =\sum_{u=1}^{U} \sum_{g=1}^{G} p_{u g} \log p_{u g}-\sum_{u=1}^{U} \sum_{g=1}^{G} p_{u g} \log p_{u \cdot}-\sum_{u=1}^{U} \sum_{g=1}^{G} p_{u g} \log p_{\cdot g} \\
& =\sum_{u=1}^{U} \sum_{g=1}^{G} p_{u g} \log p_{u g}-\sum_{u=1}^{U} \log p_{u} \cdot \sum_{g=1}^{G} p_{u g}-\sum_{g=1}^{G} \log p_{\cdot g} \sum_{u=1}^{U} p_{u g} \\
& =\sum_{u=1}^{U} \sum_{g=1}^{G} p_{u g} \log p_{u g}-\sum_{u=1}^{U} p_{u \cdot} \log p_{u \cdot}-\sum_{g=1}^{G} p_{\cdot g} \log p_{\cdot g} \\
& =\mathrm{E}\left(\mathbf{p}_{u \cdot}\right)+\mathrm{E}\left(\mathbf{p}_{\cdot g}\right)-\mathrm{E}\left(\mathbf{p}_{u g}\right)
\end{aligned}
$$

Next, show that the form that is written as weighted local segregation indices (the "divergence" form) is identical to $M$ by making use of the identity $p_{g \mid u}=p_{u g} / p_{u}$.

$$
\begin{aligned}
M & =\sum_{u=1}^{U} p_{u \cdot} L_{u} \\
& =\sum_{u=1}^{U} p_{u \cdot}\left(\sum_{g=1}^{G} p_{g \mid u} \log \frac{p_{g \mid u}}{p_{\cdot g}}\right) \\
& =\sum_{u=1}^{U} p_{u \cdot}\left(\sum_{g=1}^{G} \frac{p_{u g}}{p_{u \cdot}} \log \frac{p_{u g}}{p_{\cdot g} p_{u}}\right) \\
& =\sum_{u=1}^{U} \sum_{g=1}^{G} p_{u g} \log \frac{p_{u g}}{p_{\cdot g} p_{u} .}
\end{aligned}
$$

Hence, the "Divergence Index" is just Mutual Information. Switch the meaning of $u$ and $g$ to obtain the respective identity in the form of weighted local segregation scores for $g$.

Next, show that the form that is expressed as differences in entropy is equal to $M$ 
by making use of the first proof in this section:

$$
\begin{aligned}
M & =\sum_{u=1}^{U} p_{u \cdot}\left(\mathrm{E}\left(\mathbf{p}_{\cdot g}\right)-\mathrm{E}\left(\mathbf{p}_{g \mid u}\right)\right) \\
& =\mathrm{E}\left(\mathbf{p}_{\cdot g}\right)+\sum_{u=1}^{U} \sum_{g=1}^{G} p_{u \cdot} \frac{p_{u g}}{p_{u}} \log \left(\frac{p_{u g}}{p_{u}}\right) \\
& =\mathrm{E}\left(\mathbf{p}_{\cdot g}\right)+\sum_{u=1}^{U} \sum_{g=1}^{G} p_{u g} \log p_{u g}-\sum_{u=1}^{U} \sum_{g=1}^{G} p_{u g} \log p_{u} . \\
& =\mathrm{E}\left(\mathbf{p}_{\cdot g}\right)-\mathrm{E}\left(\mathbf{p}_{u g}\right)-\sum_{u=1}^{U} \log p_{u \cdot} \sum_{g=1}^{G} p_{u g} \\
& =\mathrm{E}\left(\mathbf{p}_{\cdot g}\right)-\mathrm{E}\left(\mathbf{p}_{u g}\right)-\sum_{u=1}^{U} p_{u \cdot} \log p_{u \cdot} \\
& =\mathrm{E}\left(\mathbf{p}_{\cdot u}\right)+\mathrm{E}\left(\mathbf{p}_{\cdot g}\right)-\mathrm{E}\left(\mathbf{p}_{u g}\right) \\
& =\sum_{u=1}^{U} \sum_{g=1}^{G} p_{u g} \log \frac{p_{u g}}{p_{\cdot g} p_{u} .}
\end{aligned}
$$

Switch the meaning of $u$ and $g$ to obtain the respective identity in the form of weighted entropy differences for $g$.

\section{References}

Alonso-Villar, Olga, Coral Del Rio, and Carlos Gradin (2012). "The Extent of Occupational Segregation in the United States: Differences by Race, Ethnicity, and Gender: The Extent of Occupational Segregation in the United States". In: Industrial Relations: A Journal of Economy and Society 51.2, pp. 179-212. DOI: 10.1111/j.1468232X. 2012.00674.x.

Alonso-Villar, Olga and Coral del Río (2010). "Local versus Overall Segregation Measures". In: Mathematical Social Sciences 60.1, pp. 30-38. DOI: 10.1016/j . mathsocsci. 2010.03 .002$.

Charles, Camille Zubrinsky (2003). "The Dynamics of Racial Residential Segregation". In: Annual Review of Sociology 29.1, pp. 167-207. DOI: 10.1146/annurev.soc. 29 . 010202.100002 .

DiPrete, Thomas A., Thijs Bol, Christina Ciocca Eller, and Herman G. van de Werfhorst (2017). "School-to-Work Linkages in the United States, Germany, and France". In: American Journal of Sociology 122.6, pp. 1869-1938. DOI: 10.1086/691327.

Elbers, Benjamin (2021). "A Method for Studying Differences in Segregation Across Time and Space". In: Sociological Methods \& Research. DOI: 10.1177/0049124121986204. 
Elbers, Benjamin, Thijs Bol, and Thomas A DiPrete (2020). "Training Regimes and Skill Formation in France and Germany An Analysis of Change Between 1970 and 2010". In: Social Forces 99.3. DOI: 10.1093/sf/soaa037.

Fiel, Jeremy E. (2013). "Decomposing School Resegregation: Social Closure, Racial Imbalance, and Racial Isolation". In: American Sociological Review 78.5, pp. 828848. DOI: $10.1177 / 0003122413496252$.

Frankel, David M. and Oscar Volij (2011). "Measuring School Segregation". In: Journal of Economic Theory 146.1, pp. 1-38. DOI: 10.1016/j . jet.2010.10.008.

Glaeser, Edward and Jacob Vigdor (2012). The End of the Segregated Century: Racial Separation in America's Neighborhoods, 1890-2010. 66. Manhattan Institute.

Gradín, Carlos, Coral Del Río, and Olga Alonso-Villar (2015). "Occupational Segregation by Race and Ethnicity in the United States: Differences Across States". In: Regional Studies 49.10, pp. 1621-1638. DOI: 10.1080/00343404.2013.864384.

Manson, Steven, Jonathan Schroeder, David Van Riper, Tracy Kugler, and Steven Ruggles (2021). "IPUMS National Historical Geographic Information System: Version 16.0 [Dataset]". In: DOI: 10.18128/D050.V16.0.

Massey, Douglas S. and Nancy A. Denton (1988). "The Dimensions of Residential Segregation". In: Social Forces 67.2, pp. 281-315. DOI: 10.2307/2579183.

Menendian, Stephen, Arthur Gailes, and Samir Gambhir (2021). "The Roots of Structural Racism Project: Twenty-First Century Racial Residential Segregation in the United States". In: Othering $\&$ Belonging Institute. DOI: https : // belonging . berkeley.edu/roots-structural-racism.

Mora, Ricardo and Javier Ruiz-Castillo (2009). "The Invariance Properties of the Mutual Information Index of Multigroup Segregation". In: Research on Economic Inequality. Ed. by Yves Flückiger, Sean F. Reardon, and Jacques Silber. Vol. 17. Emerald Group Publishing Limited, pp. 33-53. DOI: 10.1108/S1049-2585(2009) 0000017005.

- (2011). "Entropy-Based Segregation Indices". In: Sociological Methodology 41.1, pp. 159-194. DOI: 10.1111/j.1467-9531.2011.01237.x.

Orfield, Gary, Erica D. Frankenberg, and Chungmei Lee (2002). "The Resurgence of School Segregation". In: Educational Leadership 60.4, pp. 16-20.

R Core Team (2021). R: A Language and Environment for Statistical Computing. manual. R Foundation for Statistical Computing. Vienna, Austria.

Reardon, Sean F. and Glenn Firebaugh (2002). "Measures of Multigroup Segregation". In: Sociological Methodology 32.1, pp. 33-67. DOI: 10.1111/1467-9531.00110.

Roberto, Elizabeth (2015). The Divergence Index: A Decomposable Measure of Segregation and Inequality. URL: http://arxiv.org/abs/1508.01167.

Theil, Henri and Anthony J. Finizza (1971). "A Note on the Measurement of Racial Integration of Schools by Means of Informational Concepts". In: Journal of Mathematical Sociology 1.2, pp. 187-193. DOI: 10.1080/0022250X.1971.9989795. 